\title{
Infección por Chlamydia trachomatis en pacientes de una institución de salud de Bogotá y Medellín, 2012-2015
}

\author{
Jaiberth Antonio Cardona-Arias, Luz Helena Gallego-Atehortúa y Leonardo Alberto Ríos-Osorio
}

\section{Chlamydia trachomatis infection in patients of a health institution of Bogota and Medellin, 2012-2015}

Introduction: Chlamydia trachomatis presents clinical consequences and it is barely studied in Colombia. Objective: To compare the C. trachomatis infection in Bogotá and Medellín with specific frequencies by gender and age group, between 2012-2015. Methods: Descriptive study of multiple groups, with 1,660 people in Bogotá and 1,087 in Medellin. Anti-Chlamydia trachomatis test with recombinant antigens MOMP, TARP and CPAF was applied; $100 \%$ sensitivity and $99.6 \%$ specificity. It was estimated and compared the frequency of infection by gender and age group, by hypothesis testing, confidence intervals and prevalence ratios. Results: The largest proportion were women and people between 30-39 years. The frequency of positive IgG in Bogotá was 15.6\% $(95 \% \mathrm{CI}=13.4$ to 17.8$)$ in Medellin $16.9 \%(95 \% \mathrm{CI}=13.4$ to 20.4$)$, while the frequency of positive IgM was $0 \%$ in Medellin and $0.2 \%(95 \% \mathrm{CI}=0.01-1.0)$ in Bogotá; it was higher in women. In Bogotá, the frequency was higher in younger than 30 years and lower in older than 50. Discussion: The high frequency of infection, its greater occurrence in women, the differences found in the age groups, the low number of investigations in Colombia and clinical risks associated with $C$. trachomatis, show the need to improve surveillance, screening and research in this infection.

Key words: Chlamydia trachomatis; frequency; infection; Colombia.

Palabras clave: Chlamydia trachomatis; frecuencia; infección; Colombia.

\section{Introducción}

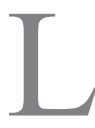

as infecciones de transmisión sexual (ITS) constituyen un problema de salud pública por ser una de las principales causas de morbilidad en el mundo, con cerca de 448 millones de casos nuevos por año, entre los 15 y 49 años; por presentar consecuencias clínicas graves y elevados costos para los sistemas de salud y los pacientes ${ }^{1,2}$. Estas incluyen diferentes agentes etiológicos con virus como el VIH y el VPH, protozoarios como Trichomonas vaginalis y bacterias como Neisseria gonorrhoeae y Chlamydia trachomatis ${ }^{3,4}$.

Chlamydia trachomatis en Colombia, según el informe sobre ITS 1976-2000, presenta una prevalencia de 6\%, con costos asociados a su manejo cercanos a US\$28 millones. Se ha asociado con graves consecuencias clínicas y epidemiológicas como enfermedad inflamatoria pélvica, complicaciones del embarazo, dolor pélvico, infertilidad y perpetuación de la transmisión por la elevada frecuencia de portadores asintomáticos; todo ello, evidencia la necesidad del diagnóstico precoz y la tamización en diferentes grupos poblacionales ${ }^{4-8}$.

En términos investigativos, son múltiples los estudios relacionados con la prevalencia de $C$. trachomatis en el ámbito mundial. En este sentido, se puede resaltar el estudio del grupo de Newman en 2012 que, con base en estudios seleccionados mediante una revisión sistemática de reportes globales de ITS, encontró una prevalencia de $4,2 \%$ (IC $95=3,7-4,7$ ) en mujeres entre 15 y 49 años 9 . Una revisión sistemática de estudios publicados entre 1980 y 2000 en mujeres asintomáticas de Europa, reportó prevalencias en un rango entre $1,7 \%$ y $17 \%$, dependiente de la población y el país de estudio ${ }^{10}$.

También se dispone de revisiones sistemáticas sobre la prevalencia de la infección en algunos países; así, en el Reino Unido, a partir de 19 estudios, se reportó la edad y el tipo de población como los principales factores que modifican la prevalencia de la infección; en este orden de ideas, bajo 20 años de edad hallaron una prevalencia de $8,1 \%$ (IC 95\% = 6,5-9,9), entre los 20-24 años 5,2\% (IC 95\% = 4,3-6,3), entre 25-29 años 2,6\% (IC 95\% = $2,0-3,3)$ y $1,4 \%$ (IC $95 \%=1,0-1,9)$ a partir de los 30 años. En función del tipo de población, la prevalencia fue $17,3 \%$ (IC 95\% = 13,6-21,8) en clínicas para problemas genito-urinarios, $12,6 \%$ (IC 95\% = 6,4-23,2) en control prenatal, $12,3 \%$ (IC 95\% = 9,8-15,3) en clínicas de atención del parto; $10,0 \%$ (IC 95\% = 8,7-11,5) en clínicas de planificación familiar y $5,0 \%$ (IC $95 \%=3,2-7,6)$ en
Universidad de Antioquia y Universidad Cooperativa de Colombia. Medellín. Facultad de Medicina, Escuela de Microbiología (JAC). Grupo de Investigación Salud y Sostenibilidad (LAR). Dinámica IPS Medellín, Colombia.

Área Asistencia (LHG).

Los autores declaran no tener conflicto de interés para la publicación del manuscrito. Financiamiento: Recursos en especie de la Universidad de Antioquia y la IPS Dinámica.

Recibido: 4 de marzo de 2016 Aceptado: 6 de septiembre de 2016 
estudios poblacionales. Finalmente, dicho estudio no halló diferencias según el tipo de prueba diagnóstica, muestra clínica ni año de realización ${ }^{11}$.

En Irán, a partir de 35 estudios hallaron una prevalencia global en mujeres de $12,3 \%$ (IC 95\% = 10,6-14,2) con rango entre $0 \%$ y $32,7 \%$; mientras que en hombres fue $10,9 \%$ (IC $95 \%=7,6-15,4 \%$ ) fluctuando entre $0 \%$ y $23,3 \%{ }^{8}$. En Australia, con base en una revisión sistemática de 40 estudios que agruparon 40.587 individuos, la prevalencia global de infección genital fue $4,6 \%$ (IC $95 \%=4,4-4,8)$, siendo levemente mayor en las mujeres; $3,3 \%$ (IC 95\% $=3,0-3,7$ ) en mujeres atendidas en clínicas de salud sexual y $5,6 \%$ (IC 95\% = 4,9-6,4\%) en jóvenes y adolescentes ${ }^{12}$.

Concretamente, en los antecedentes de investigaciones en Colombia, sólo se identificaron tres estudios a partir de una revisión de la literatura en PubMed, OVID, Scielo y Science Diretc, con las estrategias de búsqueda " $C$. trachomatis \& prevalence", "C. trachomatis \& epidemiology", "C. trachomatis \& ocurrence", "C. trachomatis \& frequency" y " $C$. trachomatis \& epidemic". El primero, en 1.829 mujeres sexualmente activas con bajos ingresos, de Bogotá, halló una prevalencia de $5 \%$, sin cambios entre quienes presentaron una citología normal $(5,0 \%)$ o alterada $(5,2 \%)^{13}$. Un segundo estudio realizado en Bogotá en 50 muestras de orina obtenidas de mujeres atendida en una Institución Prestadora de Servicios de Salud (IPS) de Bogotá por parto prematuro, reportó una prevalencia de $4 \%$ (IC 95\% 0,5-13,7) $)^{14}$. El tercer estudio en 355 muestras de orina de mujeres jóvenes halló una prevalencia de 5,3\% (IC 95\% 3,2-8,2); con 2,9\% (IC 95\% 0,9-6,5) en mujeres sin leucorrea y $7,8 \%($ IC $95 \%=4,3-12,7)$ en las pacientes con leucorrea ${ }^{15}$.

Los antecedentes expuestos evidencian la elevada prevalencia de la infección, que los estudios sobre la ocurrencia de $C$. trachomatis han sido más prolíficos en mujeres y en pacientes atendidas en clínicas, también ponen de manifiesto la baja disponibilidad de reportes simultáneos sobre la frecuencia de la infección en hombres y mujeres, así como estudios poblacionales. Concretamente en Colombia, los principales problemas con esta infección radican en que no se hace tamización ni notificación obligatoria, no existen programas de vigilancia epidemiológica o sólo se realiza vigilancia pasiva en algunas IPS, son pocos los estudios de base poblacional o que incluyan un elevado tamaño de muestra, no se dispone de publicaciones previas que comparen varias ciudades y en general, se desconoce la frecuencia de la infección y su distribución según variables como el sexo y el grupo etario.

En razón de lo anterior, el objetivo de este estudio fue comparar la infección por C. trachomatis en una IPS de Bogotá y Medellín, y sus frecuencias específicas según sexo y el grupo etario, 2012-2015.

\section{Material y Métodos}

\section{Tipo de estudio}

Descriptivo de grupos múltiples.

\section{Población}

La totalidad de personas de la IPS con medición de anticuerpos IgG e IgM en Bogotá y Medellín entre 20122015; en Bogotá 1.092 con IgG y 568 IgM, y en Medellín 473 con IgG y 614 con IgM. Estas personas cumplían los criterios de ser tamizados para C. trachomatis, en las mujeres por presentar síntomas o signos de ITS, factores de riesgo o ITS previas y los hombres por su condición de contactos, y ser residentes de la ciudad. Se excluyeron los pacientes con muestras insatisfactorias o repetidas en un año.

\section{Recolección de la información}

Se usó fuente secundaria con registros de la IPS, para controlar sesgos de información se hizo análisis de reproducibilidad de la extracción de los datos de pacientes que consultaron en un período epidemiológico (un mes) en cada año; esto se hizo en dos ocasiones (con diferencia de un mes), por un único operario y para un mismo período epidemiológico. A la base de datos se le hizo verificación contingencial y por rangos. Adicional a ello, en la fase analítica de la IPS se realiza control de calidad interno y externo, y las mediciones se realizan en equipos calibrados.

\section{Pruebas de detección}

Se aplicó la prueba de detección anti-C. trachomatis, enzimoinmuno ensayos de fase sólida para cuantificación de anticuerpos IgG e IgM en suero o plasma humano, que indican infección, independiente de la etapa de la enfermedad, y utilizan antígenos recombinantes MOMP, TARP y CPAF, los que mejoran la especificidad al discriminar $C$. trachomatis de otras especies patógenas en humanos. Las pruebas presentan sensibilidad de $100 \%$ y especificidad de $99,6 \%{ }^{16}$, lo que corrobora su validez diagnóstica para estudios de frecuencia de la infección por la baja probabilidad de resultados falsos negativo o positivos.

\section{Análisis estadístico}

La descripción de la edad se hizo con medidas de resumen y el sexo, el año y el grupo etario con frecuencias. Se estimó la frecuencia de infección según positividad para IgG e IgM en las dos ciudades, con sus intervalos de confianza de 95\%. La frecuencia de historia de infección según IgG se comparó con el año de estudio con la prueba $\chi^{2}$ de Pearson, con el sexo con la prueba $Z$ (dado el cumplimiento de la aproximación binomial a la normal) y con el grupo etario con la prueba $\chi^{2}$ de tendencia lineal. Se estimaron las frecuencias específicas de historia de 
infección según $\operatorname{IgG}$, según sexo, grupo etario y año de estudio, con sus intervalos de confianza de $95 \%$. Para las variables que presentaron relación estadística se estimaron razones de prevalencia para estimar la fuerza de la asociación. Cabe aclarar que el análisis bivariado no se realizó para la infección según positividad para IgM, dada su baja frecuencia. Tanto las frecuencias globales como las específicas se compararon entre Bogotá y Medellín a través de intervalos de confianza para la diferencia de proporciones y la prueba $\mathrm{Z}$.

Para el análisis de la confusión se evaluó el cumplimiento de los siguientes criterios: asociación entre el sexo y la infección o positividad para IgG, asociación entre el grupo etario y la infección; asociación entre sexo y grupo etario.

\section{Aspectos éticos}

Se garantizó la confidencialidad de la información manejando los registros de los pacientes con códigos numéricos, el proyecto contó con aval de la IPS la cual evaluó la pertinencia del estudio, su calidad metodológica y el cumplimiento de principios éticos de la investigación científica realizada con fuente secundaria. Se siguieron las directrices de la Declaración de Helsinki y la Resolución 8.430 del Ministerio de Salud de Colombia según la cual este estudio se clasifica como de riesgo mínimo.

\section{Resultados}

En los 1.092 pacientes con tamización de infección crónica en Bogotá se halló una edad media de 36,5 $\pm 11,8$ años, con rango intercuartil de 29 a 44 y rango entre 0,3 y 84 años; la mayor proporción de pacientes se registró en $2014(41,4 \%)$, mujeres $(72,4 \%)$ y del grupo etario entre 30 y 39 años (38,1\%). En la misma ciudad, en las 568 mediciones de posible infección aguda se halló una edad media de 33,8 $\pm 12,2$, rango entre 0,1 y 75 años, y rango intercuartil de 27,2 a 40; la mayor proporción de pacientes se encontró en el año 2013 (38,2\%), mujeres $(64,3 \%)$ y del grupo entre 30 y 39 años (42,6\%) (Tabla 1).

Por su parte, en Medellín en 473 pacientes sometidos a diagnóstico de infección crónica la edad media fue 33,7 $\pm 11,7$ años con rango entre 0,2 y 83 , y rango intercuartil de 26 a 33; con mayor proporción en el año 2014 (36,6\%) y mujeres $(65,5 \%)$. En las 614 mediciones de posible infección aguda la edad media fue 32,2 $\pm 13,0$ años, rango 0,1 a 81 y rango intercuartil de 25 a 32 ; con proporciones más altas para el año $2013(38,6 \%)$ y mujeres $(61,4 \%)$ (Tabla 1).

La frecuencia de infección según positividad para anticuerpos IgG en Bogotá fue 15,6\% (IC 95\% = 13,4-17,8) y en Medellín 16,9\% (IC 95\% = 13,4-20,4); sin hallar diferencias significativas entre ellas (Vp Prueba $Z>0,05$ ).

\begin{tabular}{|c|c|c|c|c|}
\hline & \multicolumn{2}{|c|}{ Bogotá \% (\#) } & \multicolumn{2}{|c|}{ Medellín \% (\#) } \\
\hline & $\begin{array}{c}\lg G \\
\text { (n: } 1.092)\end{array}$ & $\begin{array}{c}\lg M \\
(n: 568)\end{array}$ & $\begin{array}{c}\lg G \\
(n: 473)\end{array}$ & $\begin{array}{c}\lg M \\
(n: 614)\end{array}$ \\
\hline \multicolumn{5}{|l|}{ Año } \\
\hline 2012 & $1,0 \quad(11)$ & $0,7 \quad(4)$ & $0,2 \quad(1)$ & $0,2 \quad(1)$ \\
\hline 2013 & $28,8(314)$ & $38,2(217)$ & $35,5(168)$ & $38,6(237)$ \\
\hline 2014 & $41,4(452)$ & $36,8(209)$ & $36,6(173)$ & $32,7(201)$ \\
\hline 2015 & $28,8(315)$ & $24,3(138)$ & $27,7(131)$ & $28,5(175)$ \\
\hline \multicolumn{5}{|l|}{ Sexo } \\
\hline Mujer & $72,4(791)$ & 64,3 (365) & $65,5(310)$ & $61,4(377)$ \\
\hline Hombre & $27,6(301)$ & 35,7 (203) & 34,5 (163) & $38,6(237)$ \\
\hline \multicolumn{5}{|l|}{ Grupo etario (años) } \\
\hline Menores de 20 & $3,4 \quad(37)$ & $9,9 \quad(56)$ & $6,1 \quad(29)$ & $9,8 \quad(60)$ \\
\hline $20-29$ & $24,5(268)$ & $21,5(122)$ & $31,3(148)$ & $30,5(187)$ \\
\hline $30-39$ & $38,1(416)$ & $42,6(242)$ & $39,5(187)$ & $37,6(231)$ \\
\hline $40-49$ & $20,5(224)$ & $16,9 \quad(96)$ & $15,0 \quad(71)$ & $15,0 \quad(92)$ \\
\hline $50-59$ & $9,5(104)$ & $6,7 \quad(38)$ & $4,9 \quad(23)$ & $4,1 \quad(25)$ \\
\hline 60 o más & $3,9 \quad(43)$ & $2,5 \quad(14)$ & $3,2 \quad(15)$ & $3,1 \quad(19)$ \\
\hline \multicolumn{5}{|c|}{ Frecuencia de infección } \\
\hline Negativo & $80,2(876)$ & 99,5 (565) & 79,5 (376) & $99,7(612)$ \\
\hline Indeterminado & $4,2 \quad(46)$ & $0,4 \quad(2)$ & $3,6 \quad(17)$ & $0,3 \quad(2)$ \\
\hline Positivo & $15,6(170)$ & $0,2 \quad(1)$ & 16,9 & $0,0 \quad(0)$ \\
\hline
\end{tabular}

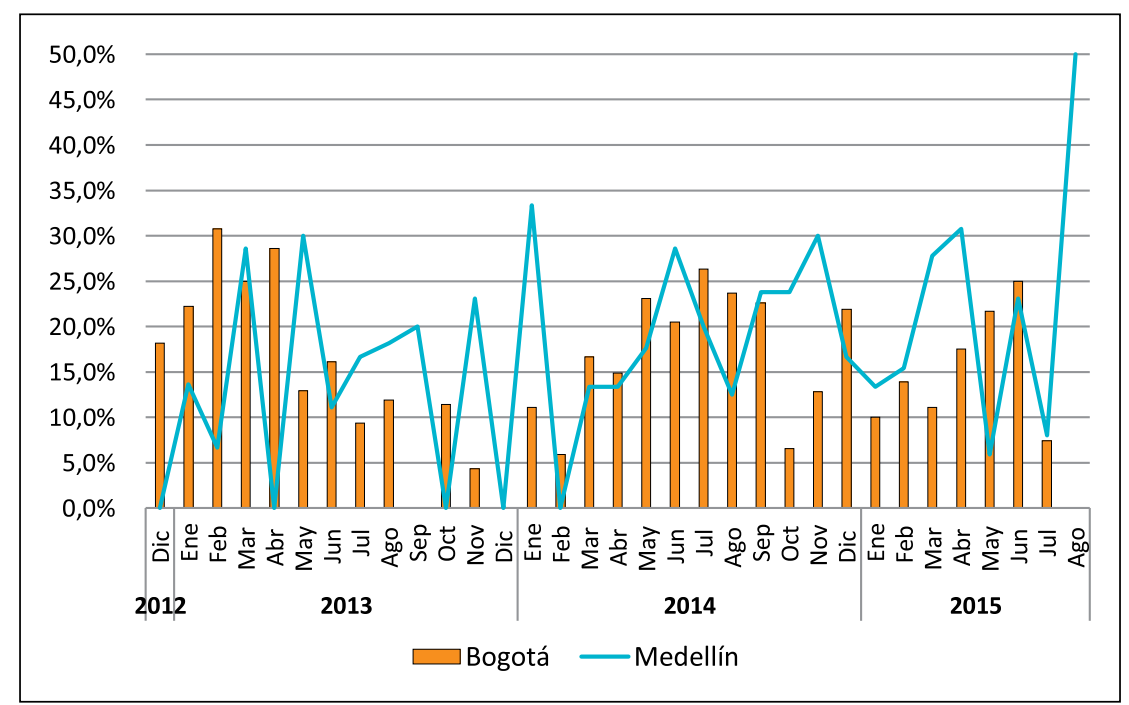

Figura 1. Frecuencia de infección (IgG positiva) por período epidemiológico en Bogotá y Medellín.

La frecuencia de infección según anticuerpos IgM en Medellín fue cero y en Bogotá 0,2\% (IC 95\% = 0,01-1,0), un único caso positivo correspondiente a una mujer de 42 años diagnosticada en agosto de 2013 (Tabla 1).

En la Figura 1 se presenta la frecuencia de infección según anticuerpos IgG en los 33 períodos epidemiológicos evaluados en ambas ciudades. Al comparar las frecuencias en los cuatro años evaluados en cada ciudad, no se 
presentó asociación estadística (Tabla 2); al tiempo que la comparación de las frecuencias específicas de cada año entre Bogotá y Medellín tampoco presentaron diferencias estadísticas (Vp Prueba $Z>0,05$ ).

Tanto en Medellín como en Bogotá se halló una frecuencia de infección según anticuerpos IgG estadísticamente mayor en las mujeres (Tabla 2); vale precisar que, al comparar los datos específicos de hombres y mujeres entre las dos ciudades, no se hallaron diferencias esta-

\begin{tabular}{|c|c|c|c|c|}
\hline & \multicolumn{2}{|c|}{ Bogotá } & \multicolumn{2}{|c|}{ Medellín } \\
\hline & $\%(\#)$ & IC $95 \%$ & $\%(\#)$ & IC $95 \%$ \\
\hline \multicolumn{5}{|l|}{ Año } \\
\hline 2012 & $18,2 \quad(2)$ & 2,$3 ; 51,8$ & $0,0 \quad(0)$ & - \\
\hline 2013 & $13,7 \quad(43)$ & 9,$7 ; 17,7$ & $13,7(23)$ & 8,$2 ; 19,2$ \\
\hline 2014 & 17,0 & 13,$5 ; 20,6$ & $18,5(32)$ & 12,$4 ; 24,6$ \\
\hline 2015 & 15,2 & 11,$1 ; 19,4$ & $19,1(25)$ & 12,$0 ; 26,2$ \\
\hline$V p \chi^{2}$ & \multicolumn{2}{|c|}{0,645} & \multicolumn{2}{|c|}{0,533} \\
\hline \multicolumn{5}{|l|}{ Sexo } \\
\hline Mujer & $18,2(144)$ & 15,$4 ; 21,0$ & $20,0(62)$ & 15,$4 ; 24,6$ \\
\hline Hombre & $8,6 \quad(26)$ & 5,$3 ; 12,0$ & $11,0(18)$ & 5,$9 ; 16,2$ \\
\hline Vp Prueba Z & \multicolumn{2}{|c|}{0,000} & \multicolumn{2}{|c|}{0,014} \\
\hline \multicolumn{5}{|l|}{ Grupo etario I (años) } \\
\hline Menores de 20 & $18,9(7)$ & 4,$9 ; 32,9$ & $10,3 \quad(3)$ & 2,$2 ; 27,3$ \\
\hline $20-29$ & $18,3(49)$ & 13,$5 ; 23,1$ & $12,2(18)$ & 6,$6 ; 17,8$ \\
\hline $30-39$ & $15,4(64)$ & 11,$8 ; 19,0$ & $20,3(38)$ & 14,$3 ; 26,4$ \\
\hline $40-49$ & $15,6(35)$ & 10,$6 ; 20,6$ & $22,5(16)$ & 12,$1 ; 33,0$ \\
\hline $50-59$ & $11,5(12)$ & 4,$9 ; 18,2$ & $17,4 \quad(4)$ & 5,$0 ; 38,8$ \\
\hline 60 o más & $7,0(3)$ & 1,$5 ; 19,1$ & $6,7 \quad(1)$ & 0,$2 ; 31,9$ \\
\hline$V p \chi^{2}$ tendencia lineal & \multicolumn{2}{|c|}{0,032} & \multicolumn{2}{|c|}{0,239} \\
\hline \multicolumn{5}{|l|}{ Grupo etario I (años) } \\
\hline Menores de 30 & $18,4(56)$ & 13,$8 ; 22,9$ & $11,9(21)$ & 6,$8 ; 16,9$ \\
\hline $30-49$ & $15,5(99)$ & 12,$6 ; 18,3$ & $20,9(54)$ & 15,$8 ; 26,1$ \\
\hline 50 o más & $10,2(15)$ & 5,$0 ; 15,4$ & $13,3 \quad(5)$ & 4,$4 ; 28,1$ \\
\hline$V p \chi^{2}$ tendencia lineal & \multicolumn{2}{|c|}{0,029} & \multicolumn{2}{|c|}{0,129} \\
\hline
\end{tabular}

Tabla 3. Medidas de asociación para la frecuencia de infección (IgG positivo) en Bogotá y Medellín, según sexo y grupo etario

\begin{tabular}{|c|c|c|c|c|}
\hline & \multicolumn{2}{|c|}{ Bogotá } & \multicolumn{2}{|c|}{ Medellín } \\
\hline & P (\#) & RP (IC 95\%) & P (\#) & RP (IC 95\%) \\
\hline \multicolumn{5}{|l|}{ Sexo } \\
\hline Mujer & $18,2(144)$ & $2,1(1,4-3,1)^{* *}$ & $20,0(62)$ & $1,81(1,1-2,9)^{* *}$ \\
\hline Hombre $^{a}$ & $8,6(26)$ & 1,0 & $11,0(18)$ & 1,0 \\
\hline \multicolumn{5}{|c|}{ Grupo etario (años) } \\
\hline$<$ de 30 años & $18,4(56)$ & $1,80(1,1-3,1)^{\star *}$ & $11,9(21)$ & $0,90(0,36-2,24)$ \\
\hline 30-49 años & $15,5(99)$ & $1,52(0,91-2,53)$ & $20,9(54)$ & $1,59(0,68-3,72)$ \\
\hline$\geq 50$ años $^{\mathrm{a}}$ & $10,2(15)$ & 1,0 & $13,3(5)$ & 1,0 \\
\hline
\end{tabular}

P: Proporción de infección. RP: Razón de Prevalencia. aCategoría de referencia. ${ }^{* *} V p<0,01$. dísticas (Vp Prueba $Z>0,05)$. En relación con la fuerza de asociación entre la infección y el sexo, en Bogotá por cada hombre con historia de infección (positivos para IgG) presentan 2,1 mujeres con la infección, mientras que en Medellín dicha infección fue $81 \%$ mayor en las mujeres (Tabla 3).

En los análisis según el grupo etario sólo se presentaron diferencias estadísticas en Bogotá, siendo más alta en las personas con menos de 30 años y más baja en los sujetos con 50 o más años (Tabla 2); cabe aclarar que al comparar las frecuencias específicas en los tres grupos etarios (menores de 30, 30-49 y 50 o más años) en Bogotá y Medellín, no se hallaron diferencias estadísticas (Vp Prueba $Z>0,05)$. En las medidas de asociación, en Bogotá la prevalencia de infección por anticuerpos IgG fue $80 \%$ mayor en los menores de 30 años frente a las personas con 50 o más años (Tabla 3 ).

En Bogotá no se halló asociación entre sexo y grupo etario (vp $\chi^{2}$ tendencia lineal $=0,143$ ), por lo que las asociaciones de la infección con estas dos variables independientes no están confundidas.

\section{Discusión}

La infección según la presencia de anticuerpos IgG en Bogotá fue $15,6 \%$ (IC 95\% = 13,4-17,8) y en Medellín $16,9 \%$ (IC $95 \%=13,4-20,4$ ); esto evidencia una ocurrencia similar en las ciudades estudiadas y supone una endemia elevada al tener presente que en los cuatro años evaluados no se hallaron diferencias estadísticas. Esto coincide con estudios previos en los que se han reportado prevalencias elevadas para diferentes poblaciones; así, en Colombia en población general es $6 \%$. Una revisión sistemática de estudios publicados entre 1980 y 2000 en mujeres asintomáticas de Europa reportó una magnitud de la infección en un rango entre $1,7 \%$ y $17 \%$, dependiente de la población y el país de estudio ${ }^{10}$.

La infección en las dos ciudades colombianas difiere de lo reportado en una revisión sistemática de Australia, en la cual fue de 3,3\% (IC 95\%=2,8-3,9) en trabajadoras sexuales y $1,6 \%$ (IC $95 \%=1,2-2,0)$ en hombres que tienen sexo con otros hombres ${ }^{12}$. Al mismo tiempo, resulta mayor que el reporte del grupo de Newman en 2012 quien halló una frecuencia del 4,2\% (IC $95=3,7-4,7$ ) en mujeres entre 15 y 49 años 9 .

A pesar de la elevada heterogeneidad en la ocurrencia de la infección, puede evidenciarse que en diversas poblaciones de comunidad general o clínicas, hasta $20 \%$ puede resultar infectado; esto corrobora la necesidad de la tamización, el diagnóstico oportuno y el tratamiento precoz, para disminuir la recurrencia de este infección y sus consecuencias clínicas; máxime al tener presente que la variabilidad en la magnitud de la infección no depende del tipo de prueba diagnóstica ni la muestra empleada ${ }^{11}$. 
En ambas ciudades, la infección fue mayor en las mujeres, lo que evidencia que este grupo presentan mayor riesgo, no sólo para la infección con C. trachomatis sino también sus efectos clínicos ${ }^{4-7}$. Esto coincide con revisiones sistemáticas que han reportado infección en $12,3 \%$ (IC $95 \%=10,6-14,2$ ) de las mujeres con rango entre 0 y $32,7 \%$; mientras que hombres $10,9 \%$ (IC 95\% $=7,6-15,4 \%$ ) con rango entre 0 y $23,3 \%{ }^{8}$. Aunque varía con respecto a los resultados de una revisión sistemática en Australia, en la cual no se documentaron diferencias significativas según el sexo, así, en hombres indígenas fue $7,5 \%$ (IC 95\% = 6,4-8,6\%), en mujeres indígenas 8,7\% (IC $95 \%=7,9-9,7 \%$ ), en hombres no indígenas $1,5 \%$ (IC $95 \%=1,1-1,9)$ y en mujeres no indígenas $1,4 \%$ (IC $95 \%=0,9-2,0 \%)^{12}$.

Se debe tener presente que, a pesar que los hombres presentan una menor frecuencia de infección y que estudios previos han documentado una baja carga de esta infección en su salud sexual y reproductiva, la evidencia sobre la relación de la infección con C. trachomatis y orquitis, epididimitis e infertilidad en población masculina, requieren un mejor seguimiento y cohortes de mayor tamaño ${ }^{6}$.

Según el grupo etario, sólo se presentaron asociaciones estadísticas en Bogotá; esto pone de manifiesto cómo la magnitud de la infección por subgrupos puede cambiar, afianzando la necesidad de conocer o describir el perfil de la infección en cada población, como base para la orientación de decisiones sanitarias y de políticas de control y atención.

En Bogotá la infección fue más alta bajo 30 años de edad y más baja en los sujetos con 50 o más años, lo que coincide con una revisión sistemática realizada en el Reino Unido en la cual hallaron una mayor ocurrencia en jóvenes, con una disminución progresiva a medida que aumentaba la edad; así, en bajo 20 años fue 8,1\% (IC 95\% = 6,5-9,9), entre 20-24 años 5,2\% (IC 95\%= $4,3-6,3$ ), entre $25-29$ años $2,6 \%$ (IC 95\% = 2,0-3,3) y a partir de los 30 años 1,4\% (IC 95\% $=1,0-1,9)^{11}$. Tanto en población general del Reino Unido como en sospechosos de la infección en Bogotá, se observa una disminución gradual de la infección en la medida que aumenta la edad $^{11}$, lo cual resulta útil para identificar a los jóvenes como un grupo de mayor riesgo para la transmisión y recurrencia de la infección. Cabe aclarar que en Medellín no se observó este gradiente, pero la frecuencia entre los 30 y 49 años fue mayor a los demás grupos etarios, permitiendo identificar el segmento de la población en mayor riesgo para la transmisión.

En otras poblaciones, como jóvenes menores de 25 años, se ha reportado una frecuencia global de 7\%; de esta población, el subgrupo de mujeres embarazadas presentó una frecuencia de $19 \%$ mientras que en no embarazadas fue $5,5 \%$. Además, dicho estudio no halló asociación por edad, número de compañeros sexuales, edad de la primera relación sexual, sintomatología urológica o ginecológica, ni estrato social ${ }^{18}$.

En relación con el diagnóstico de $C$. trachomatis, debe precisarse que éste ha avanzado ostensiblemente, en la medida que en los 60 s sólo existía en cultivo, mientras que en la actualidad se dispone de técnicas inmunológicas y moleculares, con un amplio rango de valores de validez. En este sentido, los estudios deben propender por aplicar pruebas con elevada sensibilidad y especificidad; aunque en general, la mayoría de técnicas no invasoras presentan buena utilidad diagnóstica en poblaciones sintomáticas y con alta prevalencia ${ }^{19}$.

Finalmente, debe destacarse que los estudios descriptivos, tienen la ventaja de permitir la generación de hipótesis, identificar potenciales factores de riesgo, estudiar infecciones de larga duración o curso lento y generan información útil para la planificación y administración de servicios asistenciales. Particularmente los diseños de grupos múltiples permiten comparar la infección con diversos factores de exposición en varios grupos, lo que los aproxima a un diseño analítico ${ }^{20}$.

\section{Limitaciones}

Los datos corresponden a una población con sospecha de infección, por lo que su extrapolación a población general no resulta adecuada; debido a limitaciones en el registro clínico no fue posible explorar la distribución de la infección según variables como el comportamiento sexual, co-infecciones genitales, ITS o antecedentes clínicos y obstétricos.

\section{Conclusión}

La elevada frecuencia de infección en ambas ciudades, la identificación de las mujeres como el subgrupo con mayor ocurrencia, las diferencias halladas en la distribución según el grupo etario, el bajo número de investigaciones en Colombia, así como los riesgos clínicos asociados con $C$. trachomatis, ponen de manifiesto la necesidad de mejorar la vigilancia, tamización e investigación en esta infección.

Agradecimientos. A Jenny Andrea Carmona Valencia, Coordinadora de Investigación de la IPS Dinámica por el suministro de la información, el apoyo y gestión para la realización de este estudio.

\section{Resumen}

Introducción: Chlamydia trachomatis presenta graves consecuencias clínicas y es poco estudiada en Colombia. Objetivo: Comparar la infección por C. trachomatis en Bogotá y Medellín, y sus frecuencias específicas según 
sexo y grupo etario, 2012-2015. Material y Métodos: Estudio descriptivo de grupos múltiples, con 1.660 personas de Bogotá y 1.087 de Medellín. Se aplicó serología anti-Chalmydia trachomatis con antígenos recombinantes MOMP, TARP y CPAF; sensibilidad 100\% y especificidad 99,6\% . Se estimó y comparó la infección según sexo y grupo etario, mediante pruebas de hipótesis, intervalos de confianza y razones de prevalencia. Resultados: La mayor proporción correspondió a mujeres y personas entre 30-39 años. La frecuencia de historia de infección, según IgG, en Bogotá fue 15,6\% (IC 95\% = 13,4-17,8), en Medellín 16,9\% (IC 95\% = 13,4-20,4), mientras que con IgM fue $0 \%$ en Medellín y $0,2 \%$ (IC $95 \%=0,01-1,0)$ en Bogotá; siendo mayor en las mujeres. En Bogotá, la frecuencia de infección fue más elevada bajo 30 años de edad y más baja sobre 50. Discusión: La elevada frecuencia de infección, la mayor ocurrencia en las mujeres, las diferencias halladas en los grupos etarios, el bajo número de investigaciones en Colombia y los riesgos clínicos asociados con C. trachomatis, evidencian la necesidad de mejorar la vigilancia, tamización e investigación en esta infección.

\section{Referencias bibliográficas}

1.- OMS. Guías para el tratamiento de las infecciones de transmisión sexual. Organización Mundial de la Salud; 2005. http://apps.who.int/ iris/bitstream/10665/43188/1/9243546260_spa pdf

2.- WHO. World Health Organization. Sexually transmitted infections. Fact sheet August 2011. http://www.who.int/mediacentre/factsheets/ fs $110 / \mathrm{en} /$

3.- Workowski K A, Berman S. Centers for Disease Control and Prevention (CDC). Sexually Transmitted Diseases Treatment Guidelines. MMWR Recomm Rep 2010; 59 (RR 12): $1-110$.

4.- Ministerio de Salud y Protección SocialColciencias Guía de Práctica Clínica para el abordaje sindrómico del diagnóstico y tratamiento de los pacientes con infecciones de transmisión sexual y otras infecciones del tracto genital. Bogotá. 2013. https://docs. supersalud.gov.co/PortalWeb/Comunicaciones/ GuiasPracticasClinica/guia027-2013infecciones-sexual-01-08-13.pdf

5.- Acosta J P, Rodríguez D, C. R. Situación de las enfermedades de transmisión sexual, Colombia,1976-2000. Biomédica 2002; 22 (1): 77- 88.

6.- Trei J S, Canas L C, Gould P L. Reproductive tract complications associated with Chlamydia trachomatis infection in US Air Force males within 4 years of testing. Sex Transm Dis 2008; 35 (9): 827-33.

7.- Pultorak E, Wong W, Rabins C, Mehta S D.
Economic burden of sexually transmitted infections: incidence and direct medical cost of Chlamydia, gonorrhea, and syphilis among Illinois adolescents and young adults, 2005- 2006. Sex Transm Dis 2009; 36 (10): 629-36.

8.- Ahmadi M, Mirsalehian A, Bahador A. Prevalence of genital Chlamydia trachomatis in Iran: a systematic review and meta-analysis. Pathog Glob Health. 2015; 109 (6): 290-9. doi: 10.1179/2047773215Y.0000000033.

9.- Newman L, Rowley J, Vander Hoorn S, Wijesooriya N S, Unemo M, Low N, et al. (2015) Global estimates of the prevalence and incidence of four curable sexually transmitted infections in 2012 based on systematic review and global reporting. PLoS ONE 10 (12): e0143304. doi:10.1371/journal. pone.0143304.

10.- Wilson J, Honey E, Templeton A, Paavonen J, Mardh A, Stary A, et al. A systematic review of the prevalence of Chlamydia trachomatis among European women. Hum Reprod Update. 2002; 8 (4): 385-94.

11.- Adams E, Charlett A, Edmunds W, Hughes G. Chlamydia trachomatis in the United Kingdom: a systematic review and analysis of prevalence studies. Sex Transm Infect 2004; 80: 354-362. doi: 10.1136/sti.2003.005454.

12.- Vajdic C M, Middleton M, Bowden F J, Fairley $\mathrm{C}$ K, Kaldor J M. The prevalence of genital Chlamydia trachomatis in Australia 1997-2004: a systematic review. Sex Health. 2005; 2 (3): 169-83.

13.- Molano M, Weiderpass E, Posso H, Morre S A, Ronderos M, Franceschi S. Prevalence and determinants of Chlamydia trachomatis infections in women from Bogota, Colombia. Sex Transm Infect 2003; 79: 474-8.

14.- Ruiz A, Sánchez R, Ostos O, Ángel E, Bonilla $\mathrm{H}$, Cifuentes $\mathrm{C}$, et al. Estudio piloto de prevalencia de infección por Chlamydia trachomatis detectada por PCR en mujeres con parto prematuro en el Instituto Materno Infantil de Bogotá. Rev Colomb Obstet Ginecol 2005; 56 (3): 225-30.

15.- Sánchez R, Ruiz A, Ostos O. Prevalencia de Chlamydia trachomatis detectada por reacción en cadena de la polimerasa en un grupo de mujeres jóvenes sintomáticas y asintomáticas en Bogotá, Colombia. Rev Colomb Obstet Ginecol 2006; 57 (3): 171-81.

16.- Orgetec Diagnosis. Anti-Chlamydia trachomatis IgG IgM. Mainz-Germany. 2011.

17.- Risser W L, Risser J M. The incidence of pelvic inflammatory disease in untreated women infected with Chlamydia trachomatis: a structured review. Int J STD AIDS 2007; 18 (11): 727-31.

18.- Huneeus A, Pumarino M, Schilling A, Robledo P, Bofil M. Prevalencia de Chlamydia trachomatis y Neisseria gonorrhoeae en adolescentes chilenas. Rev Med Chile 2009; 137: 1569-74.

19.- Martínez M. Diagnóstico microbiológico de Chlamydia trachomatis: estado actual de un problema. Rev Chilena Infectol 2001; 18 (4): 275-84.

20.- Cardona J. Ortodoxia y fisuras en el diseño y ejecución de estudios descriptivos. Rev MED 2015; 23 (1): 40-51. 